\title{
ESTUDO DA CONTAMINAÇÃO POR PARASITOS GASTROINTESTINAIS DE MAMÍFEROS EM HORTALIÇAS HERBÁCEAS, NO MUNICÍPIO DE GARANHUNS-PE
}

\section{STUDY OF CONTAMINATION OF GASTROINTESTINAL PARASITES OF MAMMALS IN HERBACEOUS VEGETABLES, NO MUNICIPALITY OF GARANHUNS-PE}

\author{
Natália Espíndola Oliveira ${ }^{1}$; Romário da Silva Batista ${ }^{2}$; Raquel Maria da Silva ${ }^{3}$ Gilmara \\ Mabel Santos ${ }^{4}$; Rafael Antonio Do Nascimento Ramos ${ }^{5}$.
}

DOI: https://doi.org/10.31692/978-65-991061-7-0.76-82

\begin{abstract}
RESUMO
A busca por uma alimentação saudável tem estimulado o consumo de hortaliças, principalmente in natura, porém os problemas causados pelas contaminações provenientes de parasitos gastrointestinais configuram um grave problema de saúde pública. Estes se disseminam principalmente através do uso de água e adubo contaminado com resíduos fecais humanos ou de animais, manuseio inadequado durante a colheita e pós-colheita, comercialização e/ou no preparo das hortaliças na mesa do consumidor. O presente trabalho objetivou avaliar a contaminação por enteroparasitos em alface (Lactuca sativa L.), produzidas em cinco propriedades da zona rural de Garanhuns no Agreste de Pernambuco. Para isso foram realizadas visitas, onde foi aplicado um questionário, com o intuito de conhecer a forma como são produzidas as hortaliças e se são realizadas as Boa Práticas Agrícolas (BPAs). Após a entrevista, foram realizadas coletas de 25 amostras da alface da variedade crespa, em seguida foi encaminhada ao Laboratório de Pesquisa de Sanidade e Reprodução de Ruminantes no Centro Laboratorial de Apoio a Pesquisa - CENLAG da UFRPE UAG, foram realizadas as análises através da metodologia de sedimentação espontânea após lavagem do material, seguida de centrifugação. Após as análises $100 \%$ das amostras estavam contaminados por estruturas parasitárias, sendo encontrados ovos de Ascarídeo com presença média de 49,29\% e Entamoeba spp. com 36,12\%. Foi possível inferir através dos resultados que os produtores não utilizam em suas unidades produtivas as Boas Práticas Agrícolas, fazendo uso de água contaminada na irrigação, bem como adubação orgânica sem tratamento adequado, corroborando com os resultados.
\end{abstract}

Palavras-Chave: Alface, Contaminação, Enteroparasitas.

\section{ABSTRACT}

The demand for a healthy diet has stimulated the consumption of vegetables, mainly in natura, but the problems caused by the contaminations from gastrointestinal parasites constitute a serious public health problem. These spread mainly through the use of water and fertilizer contaminated with human or animal fecal waste, improper handling during harvesting and post-harvesting, commercialization and / or the preparation of vegetables already on the consumer's table. The paper aimed to evaluate the contamination by enteroparasites in lettuce (Lactuca sativa L.) produced in five properties in Garanhuns's rural area in the dry area of Pernambuco. Thus, visits were made to apply a questionnaire looking for discover how the vegetables are produced, and if the "Good Agricultural Practices (GAPs)" have been made.

\footnotetext{
1,2 Agronomia, UFRPE - UAG, nataliaespindola.live@gmail.com, rsb.romario@ hotmail.com

${ }^{3}$ Enga Agrônoma, Mestranda no PPGPA- UFRPE, raquel.maria18@ hotmail.com

${ }^{4}$ Eng $^{\text {a }}$ Agrônoma, Professora da UFRPE-UAG, gilmabelsa@yahoo.com.br

${ }^{5}$ Médico veterinário, Professor da UFRPE-UAG, rafael.ramos@ufrpe.br
} 
After that, 25 samples of the crisp variety vegetables were collected and sent to the Research Laboratory of Sanitation and Reproduction of Ruminants at the Research Support Laboratory - CENLAG at UFRPE UAG. Spontaneous sedimentation methodology after washing the material followed by centrifugation was the methodology used to perform the analyzes.

After the analyzes, $100 \%$ of the samples were contaminated by parasitic structures, with Ascaridae eggs having an average presence of $49.29 \%$ and Entamoeba spp. with $36.12 \%$. It was possible to concluded, from the results, that the producers didn't use the Good Agricultural Practices in their productive units, using contaminated water to the irrigation, as well as organic fertilization without adequate treatment, corroborating with the results obtained.

Keywords: Lettuce, Contamination, Enteroparasites.

\section{INTRODUÇÃO}

A alface (Lactuca sativa L.) é uma planta herbácea, com ciclo anual, pertencente à família Asteraceae, sendo considerada a hortaliça folhosa mais importante na alimentação dos brasileiros (MOTA et al.,2016). Por ser consumida in natura é susceptível a contaminações de inúmeros microrganismos parasitas (SILVA et al., 2017). O parasita é caracterizado como um organismo que supre suas necessidades nutricionais através do corpo de outro organismo, o hospedeiro (LUZ et al., 2015). Doenças como as Enteroparasítoses intestinais são causadas por parasitos gastrointestinais e configuram um grave problema de saúde pública, principalmente em países em desenvolvimento, comprometendo o desenvolvimento físico e intelectual, principalmente nos jovens. Esses agentes estão principalmente associados a contaminação por consumo de alimentos mal higienizados (LUDWIG et al. 1999 Apud Pedrazzani et al. 1988).

Medidas preventivas chamadas de Boas Práticas Agrícolas (BPAs) por Maldonade et al. (2014), são recomendadas para evitar contaminações, dentre essas medidas menciona-se: A infraestrutura do local de beneficiamento de pós-colheita com galpão para higienização; galpão de armazenamento de defensivos agrícolas; utilização de produtos e ferramentas descontaminados nos cultivos; utilização de água de boa qualidade na irrigação; bem como construção de um sistema sanitário básico de descarte de material, são algumas das medidas que evitam a contaminação parasitológica das hortaliças.

Tendo em vista a carência de trabalhos científicos regionais, sobre a temática em estudo, onde a grande maioria são desenvolvidos nas regiões Sul e Sudeste, o presente trabalho teve o objetivo avaliar a contaminação por enteroparasitos em alface (Lactuca sativa L.), produzidas em cinco propriedades da zona rural de Garanhuns no Agreste de Pernambuco. 


\section{FUNDAMENTAÇÃO TEÓRICA}

Frutas e hortaliças são importantes fontes de micronutrientes e fibras, possuindo uma densidade energética e um baixo teor calórico. Por isso, a Organização Mundial da Saúde (OMS) incentiva o consumo no mundo através de campanhas como a reeducação alimentar (MELO E VILELA, 2007).

A aquisição de um produto hortícola isento de contaminantes garante os benefícios pelo sua inclusão nas dietas. O aumento de consumo da alface é favorecido pelos novos hábitos alimentares, assim como a facilidade de compra e a popularização dos plantios (SILVA et al,. 2017). Existem pelo menos quatro tipos de cultivo de alface no Brasil: em campo aberto, o convencional e o sistema orgânico; cultivo protegido, no sistema hidropônico e no solo (HENZ e SUINAGA, 2009. Apud FILGUEIRA, 2005; RESENDE et al., 2007).

Vários fatores podem influenciar na contaminação de hortaliças, podendo ocorrer desde a fase de plantio até a mesa do consumidor. As principais formas de contaminação são através da água usada na irrigação contaminada por material fecal; manipulação inadequada na colheita, pós-colheita sem higienização e utilização de material de adubação orgânica contaminados (ROCHA et al., 2008; FALAVIGNA et al., 2005; ARBOS et al., 2010).

Portanto a detecção laboratorial de parasitos em hortaliças é uma questão de saúde pública, pois fornece dados para formulação de uma conjuntura do cenário de produção bem como as condições higiênicas envolvidas na sua produção, estocagem, transporte e manuseio desses e sobre os riscos reais de contaminação para os consumidores (SILVA et al., 2017).

\section{METODOLOGIA}

O estudo foi realizado no período de fevereiro a outubro de 2018, em cinco propriedades: Sítio Flamengo, Laje, Olho d'água, na Comunidade Quilombola do Castainho e no sítio Baixa do Mondé, localizados do município de Garanhuns, no agreste meridional do estado de Pernambuco. A área da pesquisa é caracterizada por uma região de altitude média de 900 metros, temperatura média anual de $20,4^{\circ} \mathrm{C}$, umidade relativa de $81,8 \%$ e pluviosidade média anual de 874,4 mm (INMET, 2017).

Inicialmente foi realizada uma entrevista com os produtores, com o intuito de conhecer a forma como são produzidas as hortaliças, e se são realizadas as BPAs. No questionário foi abordado questões sobre a qualidade da água utilizada no cultivo, os procedimentos adotados na produção, colheita e pós colheita, higienização e beneficiamento das hortaliças.

Após a aplicação dos questionários e estudo dos resultados, foram realizadas coletas 
compostas de 25 amostras de alface da variedade crespa. As alfaces foram colhidas no período da manhã sendo acondicionadas em sacos plásticos de primeiro uso, e encaminhadas ao Laboratório de Pesquisa em Sanidade e Reprodução de Ruminantes no Centro Laboratorial de Apoio a Pesquisa - CENLAG para realização das análises parasitológicas.

No laboratório, as amostras foram lavadas em água corrente para retirar partículas de solo e sujidades superficiais. Após foram retiradas 5 folhas de cada amostra e lavadas separadamente em $250 \mathrm{~mL}$ de água destilada e com auxílio de um pincel previamente desinfetado em solução de hipoclorito de sódio a 2\%. O material da lavagem foi tamisado em gaze estéril e transferido para um cálice de vidro granulado para sedimentação por um período de 24 horas, através do método de sedimentação espontânea também conhecida como técnica de Lutz ou de Hoffman, Pons e Janer com adaptação para estudo parasitológico em hortaliças (SILVA et al., 2017).

Após esse período de tempo o líquido sobrenadante foi descartado, sendo o restante transferido $30 \mathrm{~mL}$ para tubos tipo Falcon estéreis. O material foi centrifugado a $3.000 \mathrm{rpm}$ por tempo de 1 minuto. Um volume de $100 \mu$ do sedimento foi transferido com o auxílio de uma pipeta de $20 \mu$ para 5 lâminas coradas com lugol utilizadas para exame microscópico (10x e 40x).

Os dados da pesquisa foram analisados através da estatística descritiva com o programa da Microsoft o Office Excel 2010, comparando os diferentes parasitos encontrados na alface (Lactuca sativa L.) de diferentes locais de coleta.

\section{RESULTADOS E DISCUSSÕES}

De acordo com o questionário aplicado inferiu-se que $100 \%$ dos produtores trabalham com mais de uma cultura e não utilizam BPAs como: local próprio para higienização de hortaliças, uso de água com tratamento; utilização de cercas que impeçam o acesso de animais na área de cultivo; utilização de EPIs; adubação orgânica tratada; armazenamento e transporte correto.

Após analise laboratorial, foi detectado estruturas parasitarias em $100 \%$ das áreas estudadas. Rocha (2008), pesquisando parasitos em alfaces produzidos em diferentes tipos de cultivo e comercializados em Recife-PE, também obteve resultado análagos com $100 \%$ das amostras provindas do sistema orgânico contaminadas por parasitos.

As estruturas encontradas em maior quantidade nesta pesquisa foram ovos de Ascarídeos com média de 49,29\% e Entamoeba spp. 36,12\%. Segungo Neves (2004), as hortaliças podem ser contaminadas por Ascarídeos quando é utilizada na irrigação, água 
contaminada por excrementos humanos/animais. Então esses resultados reafirmam que a água utilizada é de má qualidade. Foram encontrados ovos de Strongyloide (2,56\%), Ancylostoma (0,58\%), Estrongílidios (0,11\%) e larva de Ancylostoma (1,61\%) destribuídos nas propriedades estudadas. Esses resultados se assemelham aos de Rocha et al. (2008) e Rosa et al. (2016) em pesquisa relizada em Recife - PE e Videiras - SC respectivamente, onde eles encontraram os seguintes parasitos: Strongyloides spp., Ancylostoma spp., Estamoeba spp., Ancilostomideo, Toxocara Canis, Ascaris lumbricoides, Enterobius vermilaris, Entameba coli, Fasciola hepática, entre outros.

Larvas de Ancylostoma e Strongyloides foram encontradas em $80 \%$ das propriedades, assim como o trabalho de Belinelo e colaboradores realizaram em São Matheus - ES no ano de 2009. Já o Toxocara canis foi encontrado em $100 \%$ das propriedades, esses microorganismo são provinientes de animais como os cães e os gatos, visto sua presença na área de produção.Os parasitos Ancylotoma e Estrongylideos foram os que apresentaram as menores frequências com a média de $0,58 \%$ e $0,11 \%$ respectivamente, resultados expressos abaixo no (Gráfico 1).

GRÁFICO 1. Casos positivos de contaminação por enteroparasitos nas amostras de alfaces coletados nas propriedades: Sítio Flamengo (Propriedade I), Laje (Propriedade II), Olho d'agua (Propriedade III), Comunidade Quilombola Castainho (Propriedade IV) e Baixa de Mondé (Propriedade V), Garanhuns-PE.

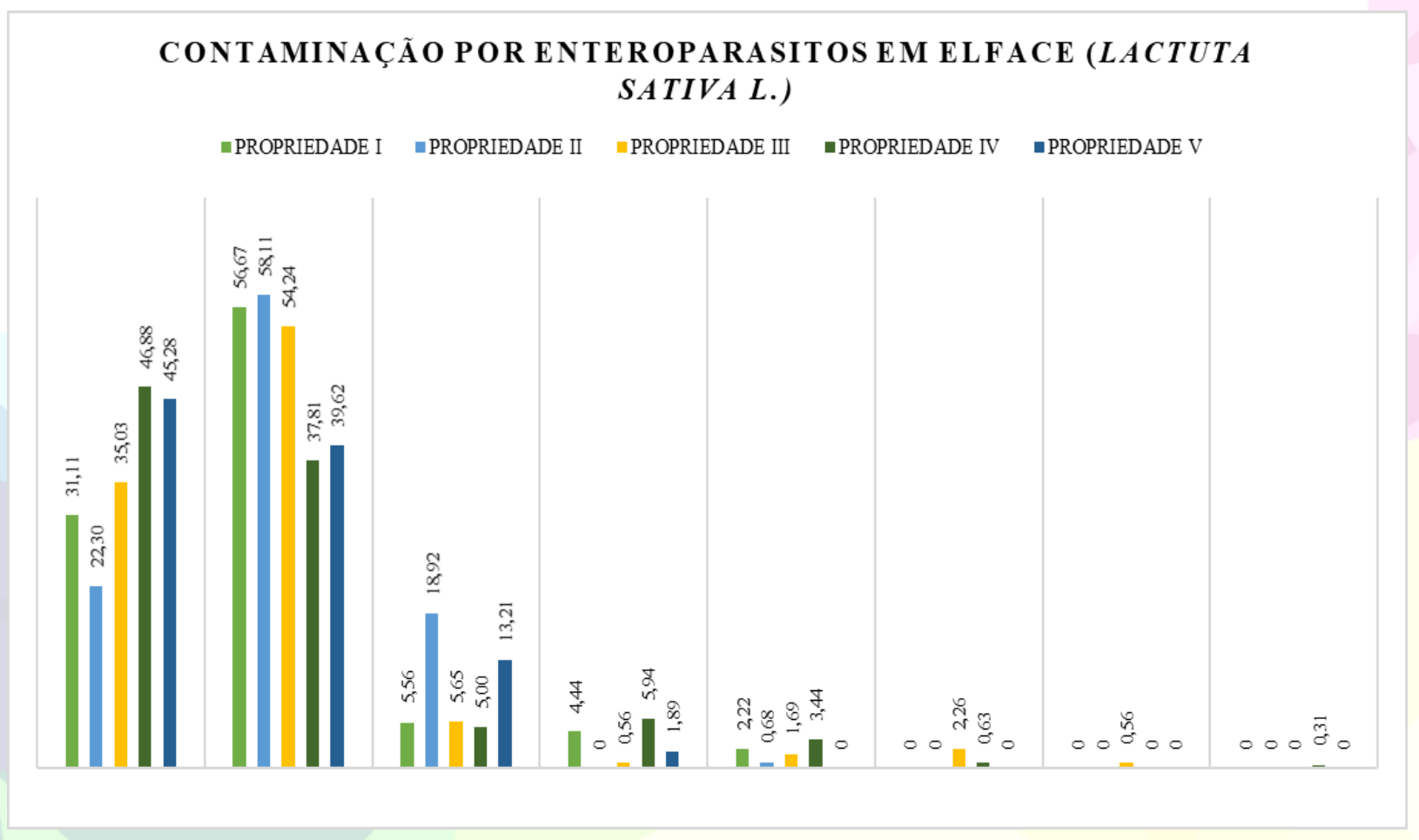


TABELA 1. Frequência de parasitos encontrados em cada propriedade pesquisada em Garanhuns-PE, 2018.

\begin{tabular}{|c|c|c|c|c|c|c|}
\hline PARASITOS & FLAMENGO & LAJE & $\begin{array}{c}\text { OLHO } \\
\text { D’ÁGUA }\end{array}$ & $\begin{array}{l}\text { COMUNIDADE } \\
\text { CASTAINHO }\end{array}$ & $\begin{array}{c}\text { BAIXA } \\
\text { DO } \\
\text { MONDÉ } \\
\end{array}$ & HOSPEDEIRO \\
\hline ENTAMOEBA SPP. & 28 & 33 & 45 & 147 & 24 & $\begin{array}{l}\text { HOMEM, } \\
\text { CANÍDEOS E } \\
\text { FELÍDEOS }\end{array}$ \\
\hline ASCARÍDEO & 51 & 86 & 51 & 115 & 21 & HOMEM \\
\hline TOXOCARA & 5 & 28 & 9 & 16 & 7 & $\begin{array}{l}\text { CANÍDEOS E } \\
\text { FELÍDEOS }\end{array}$ \\
\hline STRONGYLOIDES & 4 & 0 & 1 & 19 & 1 & HOMEM \\
\hline $\begin{array}{l}\text { LARVA DE } \\
\text { ANCYLOSTOMA }\end{array}$ & 2 & 1 & 3 & 11 & 0 & MAMÍFEROS \\
\hline ANCYLOSTOMA & 0 & 0 & 0 & 2 & 0 & MAMÍFEROS \\
\hline ESTRONGILÍDEO & 0 & 0 & 1 & 0 & 0 & EQUÍNOS \\
\hline $\begin{array}{lr}\text { LARVA } & D E \\
\text { STRONGYLOIDES }\end{array}$ & 0 & 0 & 0 & 1 & 0 & HOMEM \\
\hline
\end{tabular}

Observando a Tabela 1, percebe-se que as alfaces coletadas na propriedade da Comunidade Quilombola Castainho, apresentaram a maior frequência em detrimento das demais propriedades estudadas. Apesar das propriedades do Sítio Flamengo e Laje apresentarem menor incidência em parasitos, eles afirmam que não utilizam BPAs, isso pode ter influenciado nos resultados encontrados, aonde verifica-se que há presença principalmente de Entamoeba e Ascarídeos.

\section{Conclusões}

- Os parasitos que tiveram maiores frequências nas amostras estudadas, foram: Entamoeba Spp., Ascarídeo, Toxocara, Strongyloides, larva de Ancylostoma e os ovos de Ascarídeo e Entamoeba spp.

- A propriedade que apresentou maior contaminação foi a Comunidade do Quilombola Castainho, onde utiliza-se água de procedência duvidosa, existindo relatos que a fonte de capitação é o rio, cujo parte dos dejetos da cidade são despejados, sem tratamento.

- Todos os responsáveis legais pelas propriedades desconhecem a importância de boas práticas na produção agrícola.

- O uso das BPAs garante um alimento livre de parasitos contaminantes de mamíferos. 


\section{REFERÊNCIAS}

CARVALHO, L.E et al. Cobertura morta do solo no cultivo de alface Cv. Regina 2000, em liParaná/RO. Revista Ciência e Agrotecnologia, v.29, p.935-939, 2005.

FARIA, J.A.S et al. Estudo de alguns aspectos da disseminação de enteroparasitas na cidade de Salvador, Bahia. Estudo da poluição de águas de irrigação de hortas por cistos e ovos de enteroparasitas. Revista Baiana de Saúde Pública 13/14: 141-144, 1986/1987.

FILGUEIRA, F. A. R. Novo manual de olericultura: agrotecnologia moderna na produção e comercialização de hortaliças. 2. ed. Viçosa. MG: Ed. UFV, 2005. 412 p.

HENZ, G. P; SUINAGA, F. A. Tipos de alface cultivados no Brasil. Embrapa HortaliçasComunicado Técnico (INFOTECA-E), 2009.

LUDWIG, K. M et al. Correlação entre condições de saneamento básico e parasitoses intestinais na população de Assis, Estado de São Paulo. Revista da Sociedade Brasileira de Medicina Tropical, 547-555. 1999.

MALDONADE, I. R. Manual de boas práticas na produção de Alface / Iriani Rodrigues Maldonade [et al...]. - Brasília, DF: Embrapa Hortaliças, 2014. 44 p. - (Documentos / Embrapa Hortaliças, ISSN 1677-2229.

MELO, P.C.T; VILELA, N. J. Importância da cadeia produtiva brasileira de hortaliças. 2007. MOTA, J. H. et al. Nutrição e adubação da cultura da batata-doce. Embrapa SemiáridoCapítulo em livro científico (ALICE), 2016.

Pedrazzani ES, Mello DA, Pripas S, Fucci M, Barbosa CAA, Santoro MCM. Helmintoses intestinais. IIPrevalência e correlação com renda, tamanho da família, anemia e estado nutricional. Revista de Saúde Publica 22:384-389, 1988.

RESENDE, F. V et al. V. Cultivo de alface em sistema orgânico de produção. Brasília, DF: Embrapa Hortaliças, 2007. 16 p.

ROCHA, A. A. M; BARBOSA, R. C. S. Strongyloides spp e outros parasitos encontrados em alfaces (Lactuca sativa). Revista de Patologia Tropical, v. 37, n. 2, p. 151-160, 2008.ARBOS, Kettelin Aparecida et al. Segurança alimentar de hortaliças orgânicas: aspectos sanitários e nutricionais. Ciência e Tecnologia de Alimentos, v. 30, n. 1, 2010.

SILVA, M. V et al. Estudo parasitológico de alface (Lactuca sativa L.) em alimentos fast food comercializados em festas populares do Carirn. Biota Amazônia. Macapá, v. 7, n. 3, p. 28-32, 2017. Disponível em: Disponível em http://periodicos.unifap.br/index.php/biota. Acesso em 24 de out 2018. 